D. E. Bonds $\cdot$ N. Lasser $\cdot$ L. Qi • R. Brzyski $\cdot$ B. Caan •

G. Heiss · M. C. Limacher · J. H. Liu - E. Mason •

A. Oberman • M. J. O'Sullivan • L. S. Phillips •

R. J. Prineas $\cdot$ L. Tinker

\title{
The effect of conjugated equine oestrogen on diabetes incidence: The Women's Health Initiative randomised trial
}

Received: 24 June 2005 / Accepted: 25 October 2005 / Published online: 27 January 2006

(C) Springer-Verlag 2006

\begin{abstract}
Aims/hypothesis: Recent clinical trials have found that the combination of conjugated equine oestrogen (CEO) and medroxyprogesterone has a protective effect on the incidence of type 2 diabetes. To determine the effect of CEO alone on the incidence of diabetes mellitus in postmenopausal women, we analysed the results of the Women's Health Initiative oestrogen-alone trial. Methods: The Women's Health Initiative is a randomised, double-masked trial comparing the effect of daily $0.625 \mathrm{mg} \mathrm{CEO}$ with placebo during 7.1 years of follow-
\end{abstract}

Electronic Supplementary Material Supplementary material is available for this article at $\mathrm{http}: / / \mathrm{dx}$.doi.org/10.1007/s00125-0050096-0

D. E. Bonds $(\bowtie) \cdot$ R. J. Prineas

Section of Epidemiology, Department of Public Health Sciences,

Wake Forest University, School of Medicine,

Medical Center Boulevard,

Winston-Salem, NC 27104, USA

e-mail: dbonds@wfubmc.edu

Tel.: +336-716-6012

Fax: +336-713-4300

N. Lasser

University of Medicine and Dentistry of New Jersey (UMDNJ),

New Jersey Medical School,

Newark, NJ, USA

L. Qi · L. Tinker

Public Health Division,

Fred Hutchinson Cancer Research Center,

Seattle, WA, USA

R. Brzyski

UTHSCSA, Department of Obstetrics and Gynecology,

San Antonio, TX, USA

B. Caan

Kaiser Foundation Research Institute,

Oakland, CA, USA

G. Heiss

Department of Epidemiology, School of Public Health,

University of North Carolina at Chapel Hill,

NC, USA up of 10,739 postmenopausal women who were aged 50 79 years and had previously had a hysterectomy. Diabetes incidence was ascertained by self-report of treatment with insulin or oral hypoglycaemic medication. Fasting glucose, insulin and lipoproteins were measured in an $8.6 \%$ random sample of study participants, at baseline and at 1,3 and 6 years. Results: The cumulative incidence of treated diabetes was $8.3 \%$ in the oestrogen-alone group and $9.3 \%$ in the placebo group (hazard ratio $0.88,95 \%$ CI $0.77-1.01, p=0.072$ ). During the first year of follow-

M. C. Limacher

Division of Cardiovascular Medicine, University of Florida,

Gainesville, FL, USA

J. H. Liu

Department of Obstetrics and Gynecology,

University Hospitals of Cleveland,

Cleveland, OH, USA

J. H. Liu

Department of Reproductive Biology,

Case Western Reserve University,

Cleveland, $\mathrm{OH}$, USA

E. Mason

Department of Obstetrics and Gynecology,

John H. Storger Jr Hospital of Cook County,

Chicago, IL, USA

A. Oberman

Division of Preventive Medicine, Department of Medicine,

University of Alabama at Birmingham School of Medicine,

Birmingham, AL, USA

M. J. O'Sullivan

Department of Obstetrics and Gynecology,

University of Miami,

Miami, FL, USA

L. S. Phillips

Division of Endocrinology, Emory University,

Atlanta, GA, USA 
up, a significant fall in insulin resistance (homeostasis model assessment of insulin resistance) in actively treated women compared with the control subjects (Year 1 baseline between-group difference -0.53) was seen. However, there was no difference in insulin resistance at the 3- or 6-year follow-up. Conclusions/interpretation: Postmenopausal therapy with oestrogen alone may reduce the incidence of treated diabetes. The effect is smaller than that seen with oestrogen plus progestin. CEO should not, however, be used with the intention of preventing diabetes, as its well-described adverse effects preclude long-term use for primary prevention.

Keywords Conjugated equine oestrogen - Diabetes mellitus $\cdot$ Postmenopausal $\cdot$ Randomised double-masked trial

Abbreviations CEO: conjugated equine oestrogen HERS: Heart and Oestrogen/Progestin Replacement Study - HOMA-IR: Homeostasis model assessment of insulin resistance $\cdot \mathrm{HR}$ : hazard ratio $\cdot \mathrm{O}+\mathrm{P}$ : oestrogen plus progesterone $\cdot$ SERM: selective oestrogen receptor modifier · WHI: Women's Health Initiative

\section{Introduction}

Recent studies have suggested that postmenopausal hormone use is associated with a lower incidence of diabetes. Over the 4 years of the Heart and Oestrogen/ Progestin Replacement Study (HERS), the incidence of diabetes was $6.2 \%$ in the oestrogen plus progestin group and $9.5 \%$ in the placebo group (hazard ratio $[\mathrm{HR}]=0.65$, 95\% CI 0.48-0.89) [1]. The Women's Health Initiative (WHI) Oestrogen plus Progesterone $(\mathrm{O}+\mathrm{P})$ trial showed a similar protective effect [2]. After an average of 5.6 years of follow-up, the cumulative incidence of diabetes was $3.5 \%$ in the $\mathrm{O}+\mathrm{P}$ group and $4.2 \%$ in the placebo group, which represented a $21 \%$ relative reduction in the risk of incident treated diabetes $(\mathrm{HR}=0.79$, nominal 95\% CI $0.67-0.93)$.

The effect of oestrogen and progestin individually has not been fully elucidated. A few studies have shown that medroxyprogesterone may have deleterious effects on glucose metabolism and diabetes incidence [3, 4]. For example, a study of Navajo women who used depot medroxyprogesterone intramuscularly found that users were more likely to develop diabetes than patients who had used any type of oral combination oestrogen-progestin contraception (odds ratio $=3.8,95 \%$ CI $1.8-7.9$ ) [3]. Unopposed oestrogen, recommended only for women without a uterus, has been shown in observational studies to reduce the risk of developing type 2 diabetes. Over a decade ago, Manson and colleagues reported a reduced risk after following postmenopausal women enrolled in the Nurses' Health Study for 12 years [5]. More recently, Rossi and colleagues followed 673 postmenopausal women for an average of 3.7 years and found reduced incidence of diabetes among those who wore an oestrogen patch compared with women who took no hormones [6]. These findings suggest that use of a progestogen might increase the risk of diabetes, mitigating the beneficial effect of oestrogen on diabetes incidence. To determine if postmenopausal hormone therapy with oestrogen alone confers protection against diabetes and whether the effect is greater or less than that conferred by oestrogen plus progestin in combination, data from the recently concluded WHI oestrogen-alone trial were analysed.

\section{Methods}

Study design and population

The eligibility criteria, recruitment methods, study population characteristics, hormone regimens, randomisation, blinding, follow-up and main outcomes of the WHI oestrogen-alone trial have been previously published [7-9]. Briefly, 10,739 postmenopausal women who were aged 50-79 years and had undergone hysterectomy were recruited at 40 clinical centres throughout the USA. The primary outcome of the hormone trials was CHD, with other cardiovascular disease, breast and gynaecological cancers and hip fracture as pre-specified secondary outcomes [10]. Diabetes was a predetermined outcome measure. Major exclusion criteria were a history of previous breast cancer, any cancer within the previous 10 years except non-melanoma skin cancer, current use of corticosteroids, anticoagulants, tamoxifen or other selective oestrogen receptor modifiers (SERMs), and triglycerides $>4.56 \mathrm{mmol} / 1$. A history of venous thromboembolism was added as an exclusion criterion in 1997. Women who were unwilling to discontinue the use of menopausal hormone therapy were excluded, and a 3-month washout period was required for women who were current hormone users at the initial screening visit.

In addition to the oestrogen-alone trial, WHI also included three other randomised trials: a companion hormone trial of $\mathrm{O}+\mathrm{P}$ in women who had an intact uterus (stopped in 2002) [11], a dietary modification trial examining the health outcomes of a low-fat eating pattern, and a trial of calcium and vitamin D supplementation [7]. Approximately $29 \%$ of women in the conjugated equine oestrogen (CEO) trial also participated in the dietary modification trial, where they were randomly assigned to participate in a low-fat dietary change group or continue their usual diet. Some $58 \%$ of participants in the CEO trial also participated in a trial of calcium plus vitamin D supplementation [7]. The study protocol and consent forms were approved by the institutional review board for each participating institution. Informed consent was obtained from all participants in the trial. The intervention portion of the oestrogen-alone trial was stopped early by the National Institutes of Health.

At baseline, participants were asked if a physician had ever told them they had 'sugar diabetes' when they were 
not pregnant. Women who responded positively were considered to have self-reported diabetes at baseline and were excluded from further analysis. No medical record confirmation of self-reported diabetes was available.

\section{Randomisation, intervention and blinding}

A randomised permuted block algorithm, stratified by clinical centre site and age, was developed at the WHI Clinical Coordinating Center and implemented locally through a distributed study database. The study participants were provided CEO, $0.625 \mathrm{mg}$ daily (Premarin; Wyeth Ayerst, Philadelphia, PA, USA), or a matching placebo. Participants, clinic staff, investigators and outcomes adjudicators were blinded to treatment assignment. Neither the clinic gynaecologist nor any of the staff or investigators involved with the clinical care of the participants was involved with study outcomes assessments.

If participants developed symptoms such as breast tenderness, flexibility in the dosage of oestrogen was allowed by reducing the number of days that study medication was taken. Permanent discontinuation of study medication was required for women who developed breast cancer, venous thromboembolism, meningioma, malignant melanoma or triglyceride levels $>11.3 \mathrm{mmol} / \mathrm{l}$, or who were prescribed oestrogen, testosterone or SERMs by their personal physicians. These participants were included in the primary intention-to-treat analysis presented here.

\section{Data collection, follow-up and outcomes ascertainment}

Volunteers completed three pre-randomisation screening visits during which baseline information was obtained. Height, weight, waist and hip circumferences, and blood pressure were measured by certified staff using standardised procedures [12]. Follow-up for clinical events occurred every 6 months. During the required annual clinic visits, anthropometric and blood pressure measurements were repeated, and adherence to study medication was assessed by pill counts. A standardised written protocol, centralised training of local clinic staff, local quality assurance activities, and periodic quality assurance oversight visits by the Clinical Coordinating Centre were used to maintain uniform data collection procedures at all study sites.

All women had blood drawn after an overnight 8-h fast at baseline and at the first annual visit. Serum and plasma samples were frozen at $-70^{\circ} \mathrm{C}$ and shipped to the WHI central storage facility. A randomly selected paired (CEO and placebo) $8.6 \%$ sample of blood specimens was analysed at both time points for a set of 'core analytes' including glucose (serum), insulin (serum) and lipoproteins (EDTA-plasma). The random sampling procedure was stratified by age, clinical centre, hysterectomy status and ethnicity to oversample minority women. The $8.6 \%$ sample cohort also had fasting blood drawn using the same protocol at the third, sixth and ninth annual visits (ninth annual visit blood not yet analysed). Insulin resistance was calculated from fasting glucose and insulin according to the homeostasis model assessment of insulin resistance (HOMA-IR) model: insulin/(22.5 exp[- $\ln ($ glucose/18)]), where the units for insulin are $\mu \mathrm{U} / \mathrm{ml}$ and units for glucose are $\mathrm{mmol} / \mathrm{l}$ [13]. A subject met the criteria for metabolic syndrome if at least three of the following conditions were met: a waist circumference $>88 \mathrm{~cm}$; triglycerides $\geq 1.69 \mathrm{mmol} / 1$; HDL levels $<1.28 \mathrm{mmol} / 1$; glucose levels $>6.1 \mathrm{mmol} / 1$; systolic blood pressure $\geq 130 \mathrm{mmHg}$; or diastolic blood pressure $\geq 85 \mathrm{mmHg}$.

At each semi-annual contact, participants were asked, 'Since the date given on the front of this form, has a doctor prescribed any of the following pills or treatments?' Choices included 'pills for diabetes' and 'insulin shots for diabetes'. Thus, incident diabetes was defined as a self-report of a new physician diagnosis of diabetes treated with oral hypoglycaemic agents or insulin.

\section{Statistical analysis}

Glucose, insulin and lipoproteins were analysed on stored blood samples from 1,046 women at baseline, 909 women at Year 1 and 813 women at Year 3. Baseline variables were compared with either $\chi^{2}$ or Fisher's exact tests for categorical variables or two-sample $t$ tests for continuous variables. $p \leq 0.05$ was considered significant. Mean differences (at baseline and over time on study) in blood analytes, as well as blood pressure, BMI and waist circumference measures by treatment arm were assessed with $t$ tests. Statistical analyses of blood analyte variables were weighted by race and/or ethnicity. The incidence of diabetes was assessed with a Cox proportional hazards model, stratified by age and dietary modification trial randomisation assignment (not randomised, randomised to intervention group, randomised to control group), in which time to a first self-report of treated diabetes was the outcome variable. A sensitivity analysis was performed to account for treatment non-adherence by comparing Kaplan-Meier cumulative hazard curves for incidence of diabetes for both an intention-to-treat analysis and with participants censored 6 months after becoming non-adherent (defined as taking $<80 \%$ of study medication or starting openlabel hormone therapy).

Interactions between nine baseline characteristics (age, race/ethnicity, BMI, waist circumference, hypertension, smoking, alcohol use, physical activity and metabolic syndrome) and treatment assignment were assessed in Cox proportional hazards models that included both the risk factor (where applicable, as a continuous variable for computing the $p$ value) and randomisation assignment as main effects. Characteristics known to influence or be associated with diabetes were selected. $p$ values for assessing possible interactions were computed from likelihood ratio tests, comparing models with and without the interaction term. Results from all nine comparisons are presented. 
Table 1 Baseline characteristics of non-diabetic women randomised to oestrogen-alone or placebo in the WHI $(n=9,712)^{\mathrm{a}}$

\begin{tabular}{|c|c|c|c|c|c|}
\hline & \multicolumn{2}{|c|}{ CEO $(n=4,806)$} & \multicolumn{2}{|c|}{ Placebo $(n=4,906)$} & $p$ value \\
\hline Age group at screening (years) & - & - & - & - & 0.81 \\
\hline $50-59$ & 1,504 & 31.3 & 1,542 & 31.4 & - \\
\hline $60-69$ & 2,138 & 44.5 & 2,203 & 44.9 & - \\
\hline $70-79$ & 1,164 & 24.2 & 1,161 & 23.7 & - \\
\hline Black & 660 & 13.7 & 713 & 14.5 & - \\
\hline Hispanic & 280 & 5.8 & 295 & 6.0 & - \\
\hline Asian/Pacific Islander & 37 & 0.8 & 24 & 0.5 & - \\
\hline American Indian & 75 & 1.6 & 67 & 1.4 & - \\
\hline Unknown & 67 & 1.4 & 64 & 1.3 & - \\
\hline Duration of prior hormone use (years) & - & - & - & - & 0.83 \\
\hline$<5$ & 1,241 & 52.9 & 1,278 & 52.7 & - \\
\hline $5-10$ & 435 & 18.5 & 466 & 19.2 & - \\
\hline$>10$ & 670 & 28.6 & 682 & 28.1 & - \\
\hline BMI $\left(\mathrm{kg} / \mathrm{m}^{2}\right)$ & - & - & - & - & 0.47 \\
\hline$<25$ & 1,073 & 22.4 & 1,046 & 21.5 & - \\
\hline $25-30$ & 1,677 & 35.1 & 1,749 & 35.9 & - \\
\hline$>30$ & 2,032 & 42.5 & 2,079 & 42.7 & - \\
\hline Waist circumference $(\mathrm{cm})$ & - & - & - & - & 0.49 \\
\hline$<88$ & 2,222 & 46.3 & 2,233 & 45.6 & - \\
\hline Never & 2,480 & 52.1 & 2,430 & 50.1 & - \\
\hline Past & 1,776 & 37.3 & 1,891 & 39.0 & - \\
\hline Current & 500 & 10.5 & 528 & 10.9 & - \\
\hline Alcohol use $>1$ drink/week & 1,427 & 30.0 & 1,514 & 31.1 & 0.27 \\
\hline Lipid-lowering medication use & 393 & 8.2 & 403 & 8.2 & 0.95 \\
\hline Aspirin use & 914 & 19.0 & 943 & 19.2 & 0.80 \\
\hline History of myocardial infarction & 132 & 2.7 & 132 & 2.7 & 0.87 \\
\hline History of angina & 241 & 5.0 & 234 & 4.8 & 0.58 \\
\hline History of CABG/PTCA & 96 & 2.0 & 75 & 1.6 & 0.08 \\
\hline History of stroke & 61 & 1.3 & 71 & 1.4 & 0.45 \\
\hline History of DVT or PE & 79 & 1.6 & 77 & 1.6 & 0.77 \\
\hline Metabolic syndrome, baseline ${ }^{b}$ & 178 & 35.2 & 181 & 34.2 & 0.75 \\
\hline Total expenditure from physical activity (MET) & 4,406 & $9.8(12.8)$ & 4,420 & $9.8(12.6)$ & 0.98 \\
\hline
\end{tabular}

$C A B G$ coronary artery bypass graft, $P T C A$ percutaneous transluminal coronary angioplasty, $D V T$ deep venous thrombosis, $P E$ pulmonary embolism, $M E T$ metabolic equivalent of the task

${ }^{a} 1,027$ subjects were deleted for self-reported previous diabetes

${ }^{\mathrm{b}}$ Calculated only in those with baseline blood analysis available 
Table 2 Baseline and follow-up measurements in the non-diabetic women randomised to oestrogen-alone or placebo in the WHI (measured in an $8.6 \%$ subsample)

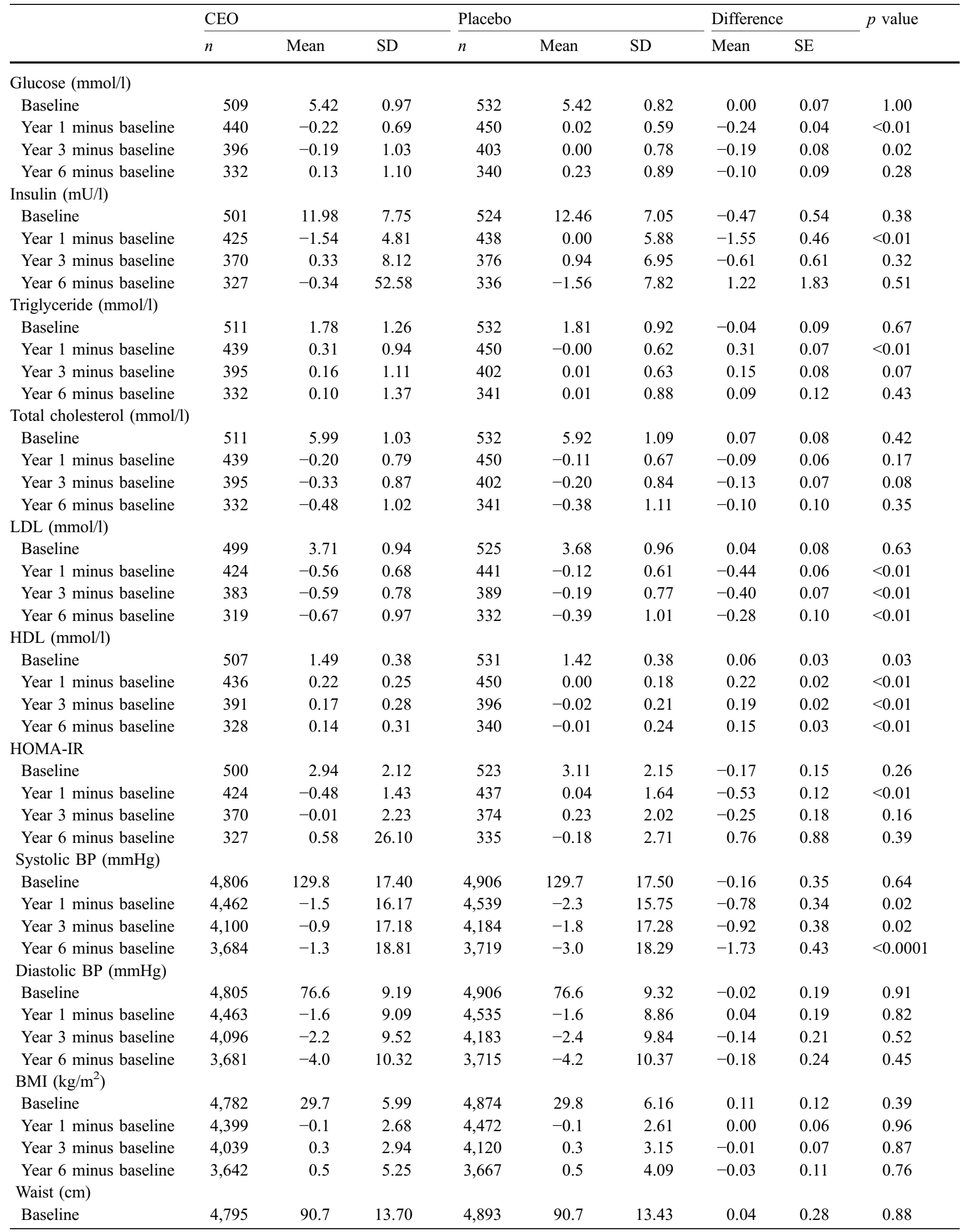


Table 2 (continued)

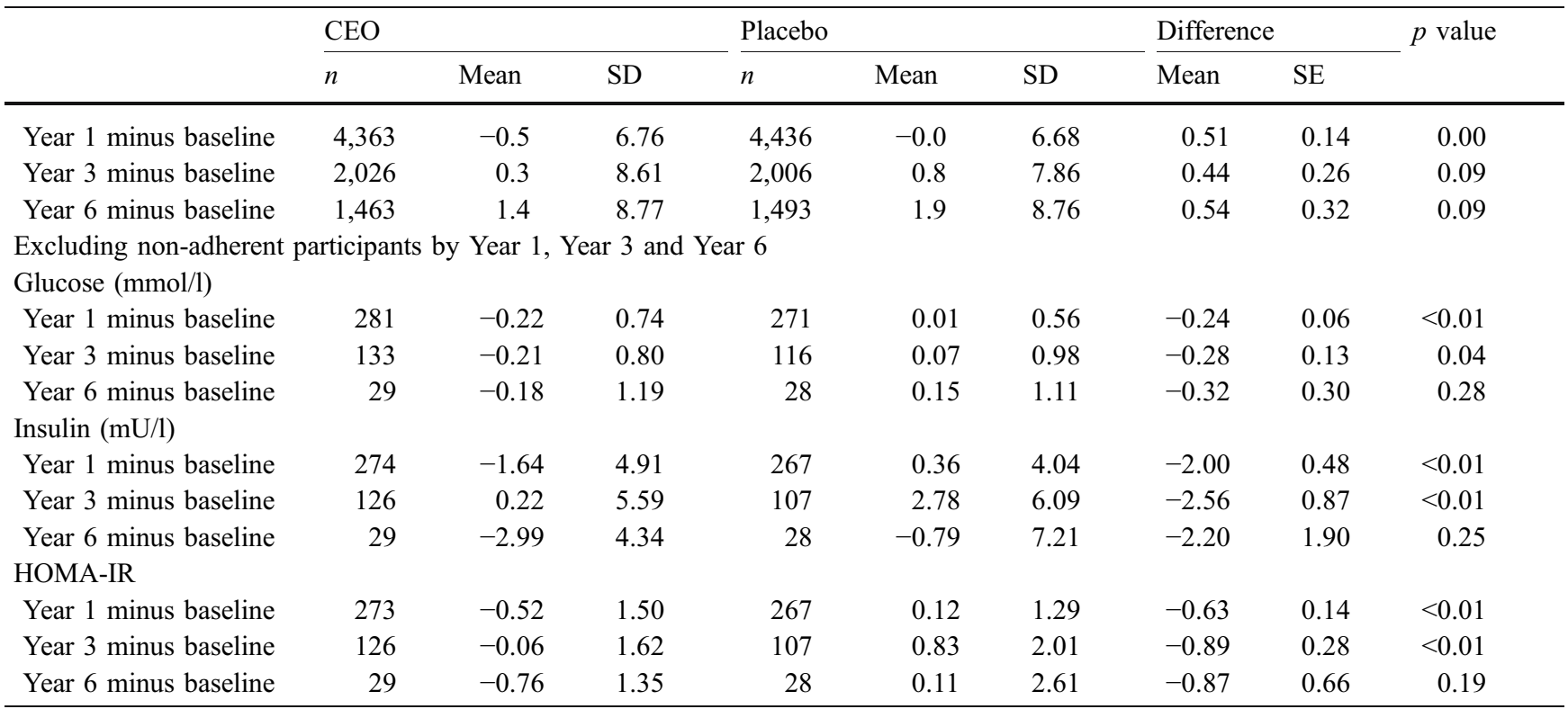

\section{Results}

Of 10,739 participants, 1,027 (504 randomised to oestrogen-alone and 523 randomised to placebo) with a reported diagnosis of diabetes at baseline were excluded from further analysis. Of the remaining 9,712 participants, 4,806 were randomised to CEO alone and 4,906 to placebo. The average age was 64 years; $76 \%$ were non-Hispanic white, $14 \%$ African American and 6\% Hispanic. Forty-three per cent had a baseline BMI $>30 \mathrm{~kg} / \mathrm{m}^{2}$ (i.e. obese) and $54 \%$ a waist circumference $>88 \mathrm{~cm}$. Although there were no differences in mean blood pressure $(130 / 77 \mathrm{mmHg})$ at baseline, treated hypertension was reported by $27 \%$; another $19 \%$ had a baseline blood pressure measurement above $140 / 90 \mathrm{mmHg}$, and $14 \%$ reported a history of hyperlipidaemia or were using cholesterol-lowering medications. The two groups were well matched at baseline (Table 1).

There were no differences in blood chemistries at baseline (Table 2). In the $8.6 \%$ subset that had baseline blood analysis, the mean plasma glucose was $5.42 \mathrm{mmol} / \mathrm{l}$ and mean insulin $12.2 \mathrm{mU} / \mathrm{l}$. Thirty-four per cent of the subsample with baseline blood analysis met the criteria for metabolic syndrome at baseline. There were also no differences in triglyceride, total cholesterol or LDL levels, but there was a small difference between the two groups in baseline HDL levels. The mean level for HDL in the oestrogen-alone participants was $1.49 \mathrm{mmol} / \mathrm{l}$, slightly higher than the mean level in the placebo participants, $1.42 \mathrm{mmol} / 1$ $(p=0.03)$.

After 1 year of study medications, blood glucose and insulin levels differed between groups (Table 2). Mean glucose decreased in the oestrogen-alone participants (Year 1 minus baseline: $-0.22 \mathrm{mmol} / \mathrm{l}$ ) while glucose levels increased in the placebo group (Year 1 minus baseline: $0.02 \mathrm{mmol} / \mathrm{l}, p<0.01$ for oestrogen-alone vs placebo com- parison). Insulin levels also decreased in the oestrogenalone group (Year 1 minus baseline: $-1.54 \mathrm{mU} / \mathrm{l}$ ) while there was no change in participants taking placebo (Year 1 minus baseline: $0.00 \mathrm{mU} / 1, p<0.01$ for oestrogen-alone vs placebo). This difference resulted in a decrease in insulin resistance measured by the HOMA model in the oestrogenalone group (Year 1 minus baseline: -0.48 ) whereas there was slight increase in the placebo group (Year 1 minus baseline: $0.04, p<0.01$ for oestrogen-alone vs placebo). At Year 3 of treatment, the glucose reduction was still present in the oestrogen-alone group (Year 3 minus baseline: $-0.19 \mathrm{mmol} / \mathrm{l}$ ) while the placebo group had no change ( $p=0.02$ for oestrogen-alone vs placebo). By Year 6 , an increase in glucose was seen in both groups (oestrogenalone Year 6 minus baseline: $0.13 \mathrm{mmol} / \mathrm{l}$ vs placebo: $0.23 \mathrm{mmol} / 1, p=0.28)$. The change in insulin was similar between the two groups at both Year 3 and Year 6 (see Table 2) resulting in no difference in insulin resistance as calculated by HOMA. While there was no difference in the change in BMI between the two treatment groups, waist circumference decreased slightly in the oestrogenalone group at 1 year (Year 1 minus baseline: $-0.5 \mathrm{~cm}$ ) while the placebo group did not change $(p<0.01$ for oestrogen-alone vs placebo). This difference was not seen at Years 3 or 6.

Nearly $54 \%$ of participants had already discontinued their study medication when the trial was stopped. Dropout and drop-in rates did not differ by study arm. If only participants adherent to study medication were considered (adherence defined as taking $\geq 80 \%$ of their medication), there was a decrease in blood glucose for the adherent oestrogen-alone group at Year 3 (Year 3 minus baseline: $-0.21 \mathrm{mmol} / \mathrm{l}$ over the 3 years of follow-up vs placebo Year 3 minus baseline: $0.07 \mathrm{mmol} / 1, p=0.04$ for oestrogen-alone vs placebo). Both groups saw increases in insulin levels (oestrogen-alone Year 3 minus baseline: $0.22 \mathrm{mU} / \mathrm{ml}$ vs 
placebo Year 3 minus baseline: $2.78 \mathrm{mU} / \mathrm{ml}, p<0.01$ for oestrogen-alone vs placebo). As a result, insulin resistance was reduced in the oestrogen-alone group (oestrogen-alone Year 3 minus baseline: -0.06 ; placebo Year 3 minus baseline: $0.83, p<0.01$ ). At Year 6 only 29 adherent active treatment and 28 adherent placebo blood samples are available, precluding meaningful comparisons.

The incidence of diabetes was lower in the participants randomised to oestrogen alone, although the difference did not attain statistical significance (Table 3 and Fig. 1). After 7.1 years of follow-up, $8.3 \%$ of women $(n=397$, annualised incidence 1.16\%) in the oestrogen-alone group reported a new diagnosis of diabetes compared with 9.3\% $(n=455$, annualised incidence $1.3 \%)$ in the placebo group $(\mathrm{HR}=0.88,95 \% \mathrm{CI} 0.77-1.01)$. Although not significant, the separation in incidence as shown in the Kaplan-Meier cumulative hazard curves is seen shortly after randomisation (Fig. 1). We examined several subgroups of participants and found no significant variation in treatment effect by age at entry, ethnicity, baseline BMI, baseline waist circumference, presence of hypertension, alcohol use, physical activity or metabolic syn-

Table 3 Self-reported diabetes incidence by treatment assignment overall and in subgroups

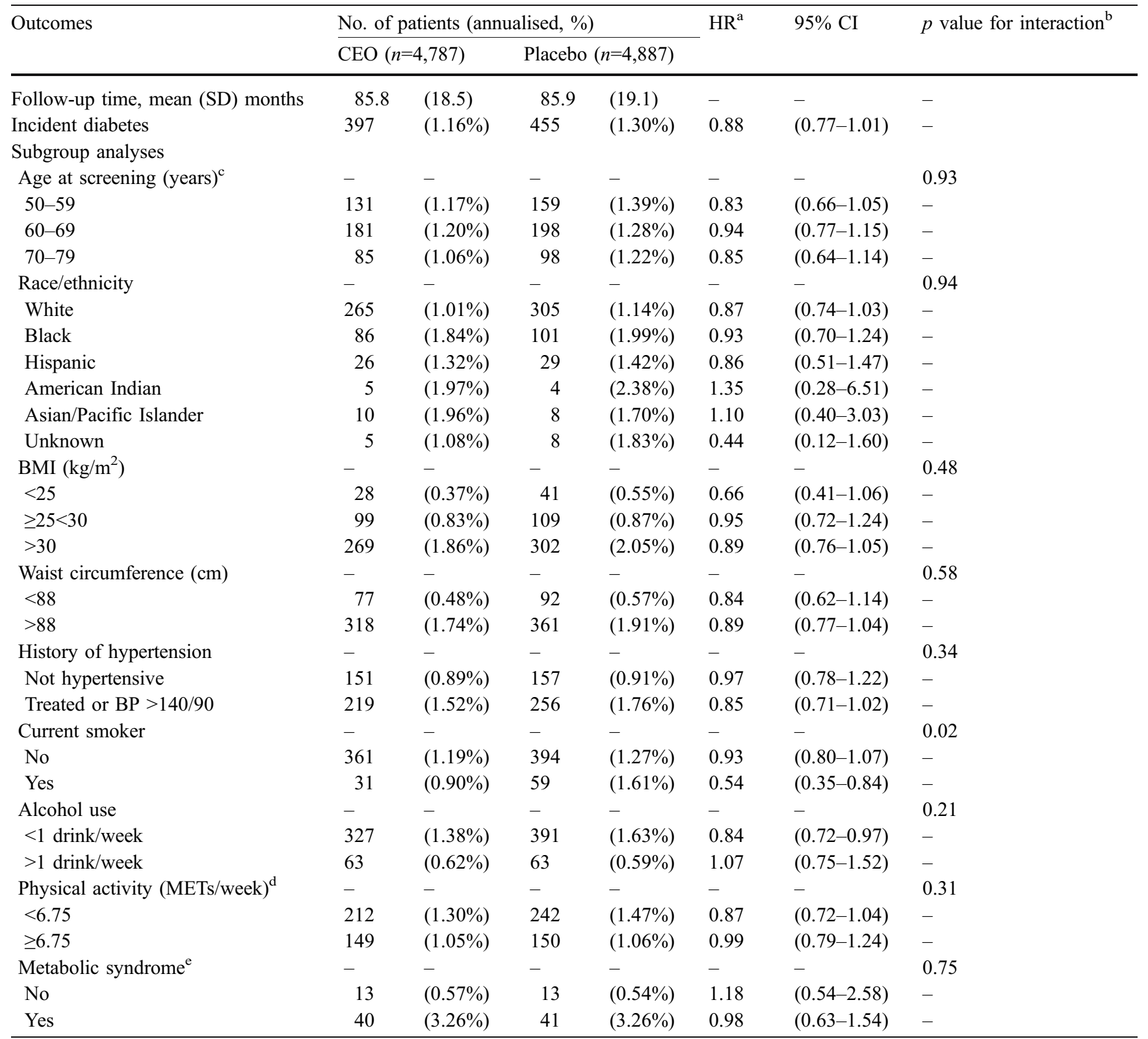

${ }^{a}$ HRs and CIs are from Cox proportional hazards analyses stratified by age and randomisation status in the low-fat-diet trial

${ }^{\mathrm{b}} p$ values from a likelihood ratio test comparing a model with the main effects for the variable of interest and intervention group vs a model with the main effects plus the interaction between the main effects

${ }^{\mathrm{c}}$ Age subgroup models are only stratified by randomisation status in the low-fat-diet trial

${ }^{\mathrm{d}}$ MET metabolic equivalent of the task

'The subsample with blood analysis was used 
Fig. 1 Diabetes incidence by treatment arm; oestrogen-alone (dashed line) vs placebo (solid line). HR $(95 \% \mathrm{CI}) 0.88$ $(0.77-1.01)$

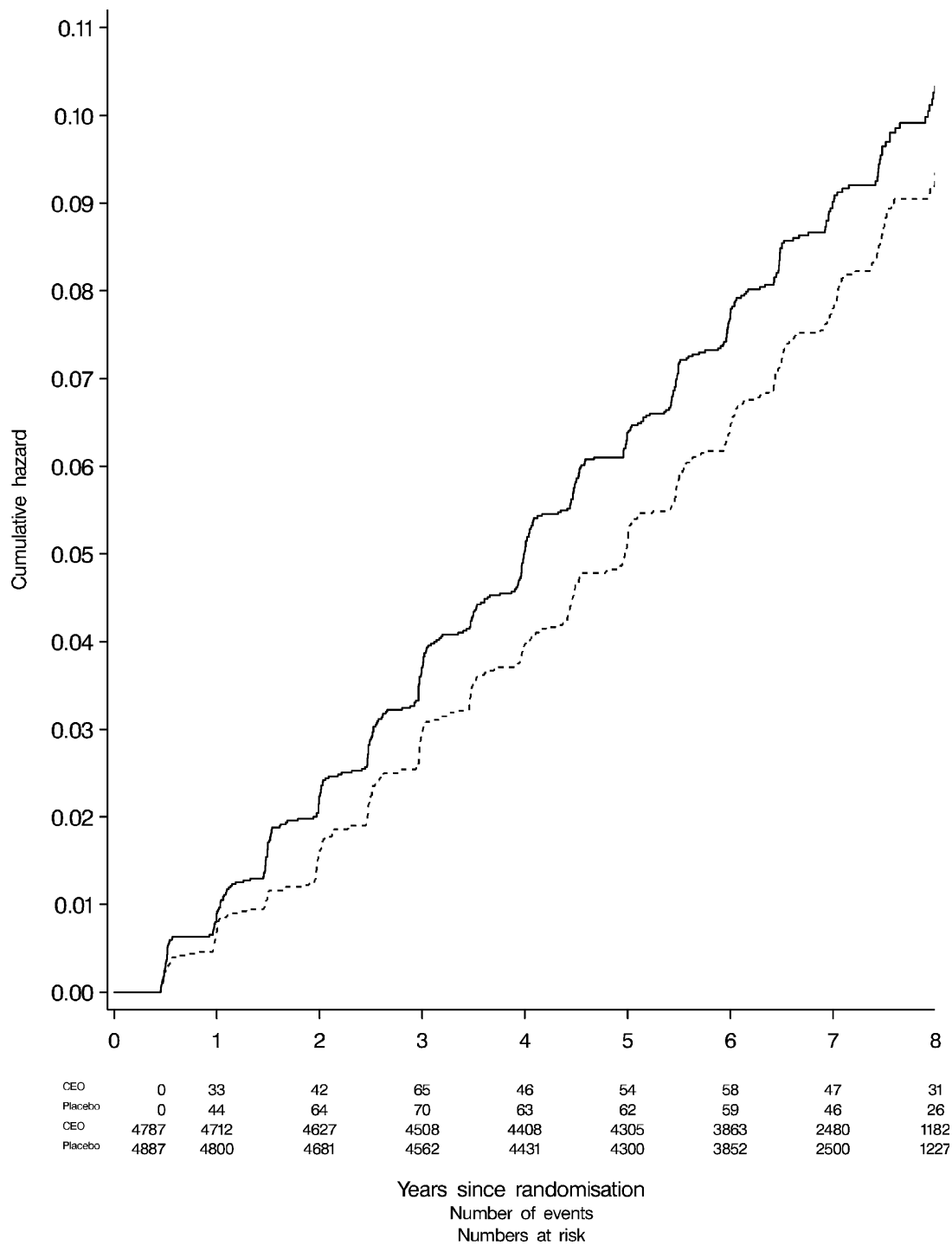

drome at baseline (Table 3). However, the treatment effect was larger for current smokers than for non-smokers (nonsmoker $\mathrm{HR}=0.93$, 95\% CI $0.8-1.07$; current smokers $\mathrm{HR}=0.54,95 \%$ CI $0.35-0.84, p$ value for smoking oestrogen-alone interaction $=0.02$ ).

When participants were censored who either took $<80 \%$ of study pills or began open-label hormone therapy, the magnitude of the difference in incident diabetes between the groups was larger, with a $27 \%$ reduction in the risk of new, treated diabetes in the women who received active hormone treatment $(\mathrm{HR}=0.73,95 \%$ CI $0.60-0.88)$.

\section{Discussion}

Our findings suggest that CEO alone given to postmenopausal women may protect against the development of diabetes. Although the difference in incidence did not quite reach nominal statistical significance, a clear trend toward a protective effect was seen with an HR of 0.88 (95\% CI 0.77-1.01). This result further supports other studies of the effect of combination hormone therapy on diabetes incidence. Both the $\mathrm{O}+\mathrm{P}$ arm of the WHI study $(\mathrm{HR}=0.79,95 \%$ CI 0.67-0.93) [2] and the HERS (HR $=0.65,95 \%$ CI $0.48-0.89)$ [1] found a significant reduction in the incidence of diabetes in women randomised to active treatment.

Blood glucose and insulin levels were significantly reduced after 1 year of CEO therapy in the WHI, but reduction did not persist at Years 3 or 6. CEO therapy also conferred a corresponding decrease in insulin resistance of 0.48 measured by the HOMA model at the end of Year 1 in the women randomised to active treatment. Similar effects on glucose and insulin have been seen in other studies. The Postmenopausal Oestrogen/Progestin Intervention Study, a placebo-controlled randomised trial of four hormone regimens included both a CEO-alone arm and CEO plus continuous medroxyprogesterone [14]. OGTT performed at baseline and 1 and 3 years showed modest reductions in both fasting glucose and insulin levels with any active therapy and a modest increase in the 2-h glucose. No significant difference by progesterone treatment group was seen. Smaller studies examining glucose and insulin levels after short-term use of CEO 
[15], oestradiol $[15,16]$ or in cross-sectional analysis [17, 18] have shown similar effects.

The process underlying the change in glucose and insulin levels with oestrogen is not fully understood, but several mechanisms have been hypothesised. Oestrogen may have a direct effect on the secretion of insulin by the pancreas. Oestrogen receptors are present in pancreatic beta cells [19], and oestrogen increases the release of insulin in beta cell culture models [20]. Oestrogen may also decrease hepatic glucose production; hypoglycaemic clamp studies showed that hepatic glucose production was lower than expected in women on hormone therapy [21]. Similar reductions have been shown in animal experiments $[22,23]$. Finally, oestrogen may reduce peripheral insulin resistance by reducing vascular reactivity in postmenopausal women with the use of both oral $[24,25]$ and transdermal oestrogen [24].

Other studies have shown an increase in glucose abnormalities and diabetes in premenopausal women using progesterone-only forms of contraception [3, 4, 26]. Given this background, a greater protective effect against diabetes was expected in the oestrogen-alone portion of the WHI trial, which was not found. We observed that the reduction in diabetes incidence reported here is less than that found in the $\mathrm{O}+\mathrm{P}$ arm of the WHI study $(\mathrm{HR}=0.79$, $95 \%$ CI $0.67-0.93)$ [2] and in the HERS trial $(\mathrm{HR}=0.65$, 95\% CI 0.48-0.89) [1].

Several possibilities may account for this difference. Most studies examining the metabolic effect of medroxyprogesterone have studied it as a contraceptive agent. When used to prevent contraception the doses of this progestin, $150 \mathrm{mg}$ depot injected every 3 months, are considerably larger than that used in the WHI, where lower circulating levels would have been anticipated [27, 28]. However, it seems unlikely that differences in medroxyprogesterone dosage could explain both risk at high dosage and benefit at lower dosage. Women in the oestrogen-alone trial were significantly different from those in the $\mathrm{O}+\mathrm{P}$ trial [29], particularly in characteristics that are associated with the development of diabetes such as age, BMI and waist circumference. This difference is demonstrated in the overall higher incidence of diabetes in the placebo group of each study arm (oestrogen-alone 9.3\%, O+P $4.2 \%$ [2]). It is possible that the higher degree of baseline risk for diabetes of the women in the oestrogen-alone trial blunted the potential protective effect of the oestrogen although no increased risk by elevated BMI was found, making this an unlikely explanation. Finally, the low dose of medroxyprogesterone used in the WHI O+P study may have potentiated the effects of CEO on insulin secretion, as seen in rat pancreatic perfusion studies [19]. Further study is needed to elucidate the separate actions and interactions of the two hormones on glucose metabolism.

There are limitations to this study. There was no confirmation of the self-reported diabetes diagnosis with medical records, nor was it possible to determine the incidence of undiagnosed diabetes. Other studies that tested the validity of self-reported diabetes have found accuracies in the range of $64-96 \%$, but none of these studies used a definition similar to that used in WHI [30-34]. Since $81.2 \%$ of participants in the WHI oestrogen-alone trial who self-reported treatment with diabetes at baseline medication were using an oral hypoglycaemic medication or insulin (unpublished data), it is unlikely that overreporting of diabetes treatment substantially affected our results. However, assessment of self-reported treated diabetes would have missed incident cases treated with lifestyle intervention and undiagnosed cases. It is unlikely that ascertainment of diabetes varied systematically by treatment arm given the masked status of the study design.

In conclusion, use of CEO alone appears to reduce the self-reported incidence of pharmacologically treated diabetes in postmenopausal women, but the overall diabetes protection from oestrogen-alone is less than that of $\mathrm{O}+\mathrm{P}$. The results of this study in conjunction with the other study results reviewed above support further investigation into the mechanism of the protective effect, examining oestrogen and progestin separately and in combination. At this time, the overall risk profile of $\mathrm{CEO}$ alone or $\mathrm{O}+\mathrm{P}$, including the increased risk for stroke, thrombotic disease and dementia, and the null effects on cardiovascular disease and breast cancer for oestrogen-alone for postmenopausal women, must be considered when weighing the possible beneficial effect on diabetes. Additionally, oestrogen-alone is licensed only for use in women who have had a hysterectomy, a small portion of the population. Given these risks, neither oestrogen-alone nor $\mathrm{O}+\mathrm{P}$ should be recommended for the primary prevention of diabetes. However, these findings may provide useful information on the aetiology of diabetes that could be used to enhance prevention programmes. If the mechanism underlying the effects on diabetes incidence can be elucidated, it may be possible to exploit this through the development of other compounds thus further expanding the range of medications available to healthcare providers.

Acknowledgement The WHI program is funded by the National Heart, Lung and Blood Institute, US Department of Health and Human Services.

Duality of interest M. C. Limacher is on a speaker's bureau for Kos Pharmaceuticals. None of the other authors has any conflicts of interest to declare.

\section{References}

1. Kanaya AM, Herrington D, Vittinghoff E et al (2003) Glycemic effects of postmenopausal hormone therapy: the Heart and Estrogen/Progestin Replacement Study. A randomized, doubleblind, placebo-controlled trial. Ann Intern Med 138:1-9

2. Margolis KL, Bonds DE, Rodabough RJ et al (2004) Effect of oestrogen plus progestin on the incidence of diabetes in postmenopausal women: results from the Women's Health Initiative Hormone Trial. Diabetologia 47:1175-1187

3. Kim C, Seidel KW, Begier EA, Kwok YS (2001) Diabetes and depot medroxyprogesterone contraception in Navajo women. Arch Intern Med 161:1766-1771 
4. Kahn HS, Curtis KM, Marchbanks PA (2003) Effects of injectable or implantable progestin-only contraceptives on insulin-glucose metabolism and diabetes risk. Diabetes Care 26:216-225

5. Manson JE, Rimm EB, Colditz GA et al (1992) A prospective study of postmenopausal estrogen therapy and subsequent incidence of non-insulin-dependent diabetes mellitus. Ann Epidemiol 2:665-673

6. Rossi R, Origliani G, Modena MG (2004) Transdermal 17-betaestradiol and risk of developing type 2 diabetes in a population of healthy, nonobese postmenopausal women. Diabetes Care 27:645-649

7. Anon (1998) Design of the Women's Health Initiative clinical trial and observational study. The Women's Health Initiative Study Group. Control Clin Trials 19:61-109

8. Hays J, Hunt JR, Hubbell FA et al (2003) The Women's Health Initiative recruitment methods and results. Ann Epidemiol 13: S18-S77

9. Anderson GL, Limacher M, Assaf AR et al (2004) Effects of conjugated equine estrogen in postmenopausal women with hysterectomy: the Women's Health Initiative randomized controlled trial. JAMA 291:1701-1712

10. Curb JD, McTiernan A, Heckbert SR et al (2003) Outcomes ascertainment and adjudication methods in the Women's Health Initiative. Ann Epidemiol 13:S122-S128

11. Rossouw JE, Anderson GL, Prentice RL et al (2002) Risks and benefits of estrogen plus progestin in healthy postmenopausal women: principal results from the Women's Health Initiative randomized controlled trial. JAMA 288:321-333

12. Anderson GL, Manson J, Wallace R et al (2003) Implementation of the Women's Health Initiative study design. Ann Epidemiol 13:S5-S17

13. Matthews DR, Hosker JP, Rudenski AS et al (1985) Homeostasis model assessment: insulin resistance and beta-cell function from fasting plasma glucose and insulin concentrations in man. Diabetologia 28:412-419

14. Espeland MA, Hogan PE, Fineberg SE et al (1998) Effect of postmenopausal hormone therapy on glucose and insulin concentrations. PEPI Investigators. Postmenopausal Estrogen/ Progestin Interventions. Diabetes Care 21:1589-1595

15. Cagnacci A, Soldani R, Carriero PL et al (1992) Effects of low doses of transdermal 17 beta-estradiol on carbohydrate metabolism in postmenopausal women. J Clin Endocrinol Metab 74: $1396-1400$

16. Zegura B, Keber I, Sebestjen M, Koenig W (2003) Double blind, randomized study of estradiol replacement therapy on markers of inflammation, coagulation and fibrinolysis. Atherosclerosis 168:123-129

17. Triusu RJ, Cowie CC, Harris MI (2000) Hormone replacement therapy and glucose metabolism. Obstet Gynecol 96:665-670

18. Nabulsi AA, Folsom AR, White A et al (1993) Association of hormone-replacement therapy with various cardiovascular risk factors in postmenopausal women. The Atherosclerosis Risk in Communities Study Investigators. N Engl J Med 328:1069-1075

19. Sutter-Dub MT (2002) Rapid non-genomic and genomic responses to progestogens, estrogens, and glucocorticoids in the endocrine pancreatic B cell, the adipocyte and other cell types. Steroids 67:77-93
20. Nadal A, Ropero AB, Fuentes E, Soria B, Ripoll C (2004) Estrogen and xenoestrogen actions on endocrine pancreas: from ion channel modulation to activation of nuclear function. Steroids 69:531-536

21. Sandoval DA, Ertl AC, Richardson MA, Tate DB, Davis SN (2003) Estrogen blunts neuroendocrine and metabolic responses to hypoglycemia. Diabetes 52:1749-1755

22. Matute ML, Kalkhoff RK (1973) Sex steroid influence on hepatic gluconeogenesis and glucogen formation. Endocrinology 92:762-768

23. Borthwick EB, Houston MP, Coughtrie MW, Burchell A (2001) The antihyperglycemic effect of estrone sulfate in genetically obese-diabetic $(o b / o b)$ mice is associated with reduced hepatic glucose-6-phosphatase. Horm Metab Res 33:721-726

24. Zegura B, Keber I, Sebestjen M, Borko E (2003) Orally and transdermally replaced estradiol improves endothelial function equally in middle-aged women after surgical menopause. Am J Obstet Gynecol 188:1291-1296

25. Vehkavaara S, Hakala-Ala-Pietila T, Virkamaki A et al (2000) Differential effects of oral and transdermal estrogen replacement therapy on endothelial function in postmenopausal women. Circulation 102:2687-2693

26. Spellacy WN, Buhi WC, Birk SA (1976) The effects of norgestrel on carbohydrate and lipid metabolism over one year. Am J Obstet Gynecol 125:984-986

27. Smit J, Botha J, McFadyen L, Beksinska M (2004) Serum medroxyprogesterone acetate levels in new and repeat users of depot medroxyprogesterone acetate at the end of the dosing interval. Contraception 69:3-7

28. Jarvinen A, Kainulainen P, Nissila M, Nikkanen H, Kela M (2004) Pharmacokinetics of estradiol valerate and medroxyprogesterone acetate in different age groups of postmenopausal women. Maturitas 47:209-217

29. Stefanick ML, Cochrane BB, Hsia J et al (2003) The Women's Health Initiative postmenopausal hormone trials: overview and baseline characteristics of participants. Ann Epidemiol 13:S78-S86

30. Kaye SA, Folsom AR, Sprafka JM, Prineas RJ, Wallace RB (1991) Increased incidence of diabetes mellitus in relation to abdominal adiposity in older women. J Clin Epidemiol 44:329-334

31. Kehoe R, Wu SY, Leske MC, Chylack LT Jr (1994) Comparing self-reported and physician-reported medical history. Am J Epidemiol 139:813-818

32. Midthjell K, Holmen J, Bjorndal A, Lund-Larsen G (1992) Is questionnaire information valid in the study of a chronic disease such as diabetes? The Nord-Trondelag diabetes study. J Epidemiol Community Health 46:537-542

33. Tretli S, Lund-Larsen PG, Foss OP (1982) Reliability of questionnaire information on cardiovascular disease and diabetes: cardiovascular disease study in Finnmark county. J Epidemiol Community Health 36:269-273

34. Kriegsman DM, Penninx BW, van Eijk JT, Boeke AJ, Deeg DJ (1996) Self-reports and general practitioner information on the presence of chronic diseases in community dwelling elderly. A study on the accuracy of patients' self-reports and on determinants of inaccuracy. J Clin Epidemiol 49:14071417 